\title{
reCHERches
}

Culture et histoire dans l'espace roman

$21 \mid 2018$

Studi per Vincenzo Consolo

\section{L'ulissismo intellettuale in Vincenzo Consolo}

\section{Giuseppe Traina}

\section{OpenEdition}

\section{Journals}

Edizione digitale

URL: https://journals.openedition.org/cher/1209

DOI: $10.4000 /$ cher.1209

ISSN: 2803-5992

\section{Editore}

Presses universitaires de Strasbourg

\section{Edizione cartacea}

Data di pubblicazione: 6 novembre 2018

Paginazione: 99-111

ISBN: 979-10-344-0027-0

ISSN: 1968-035X

Notizia bibliografica digitale

Giuseppe Traina, «L'ulissismo intellettuale in Vincenzo Consolo», reCHERches [Online], 21 | 2018, online dal 05 octobre 2021, consultato il 19 novembre 2021. URL: http://journals.openedition.org/cher/1209 ; DOI: https://doi.org/10.4000/cher.1209 


\title{
L'ulissismo intellettuale in Vincenzo Consolo
}

\author{
GIUSEPPE Traina ${ }^{1}$
}

\begin{abstract}
$\mathrm{P}^{\mathrm{s}}$ er provare a ragionare sull'ulissismo intellettuale in Vincenzo Consolo bisogna tener conto sia dei debiti di Consolo con l'Odissea come testo, sia della varia commistione fra il nostro scrittore e il protagonista dell'Odissea come personaggio che vive oltre il poema e diventa antonomastico. Si tratta di rapporti diretti, che Consolo ha esplicitato, per esempio quando ha definito l'Odissea un "grande archetipo» (Consolo 2015: 1241). Ma si tratta anche di elementi riconducibili a una nozione più ampia di ulissismo intellettuale da intendere come avventura della conoscenza oltre i confini disciplinari, temporali e geografici, di cui magari Consolo non ha lasciato tracce esplicite ma che si possono ugualmente riscontrare, pensando soprattutto alle feconde ambiguità di Ulisse, personaggio moderno non tanto perché astuto ma perché problematico portatore dell'ambivalente potere della parola ${ }^{2}$.

Consolo ha tematizzato l'ulissismo prima di tutto nel viaggio narrato in Retablo e poi in tre elementi basilari:

1. la costruzione della trilogia composta da Il sorriso dell'ignoto marinaio, Nottetempo, casa per casa e Lo Spasimo di Palermo, che evidenzia rimarchevoli elementi ulissiaci in comune, come l'azzardo e lo scacco che condannano alla morte, all'erranza o all'afasia;

2. la concezione a dittico di L'olivo e l'olivastro e Lo Spasimo di Palermo, fondata sul tema del nostos;

3. la pubblicazione di testi laterali ma ai quali Consolo mi sembra desse una certa importanza, come Il viaggio di Odisseo e soprattutto Fuga dall'Etna ${ }^{3}$.
\end{abstract}

1 Giuseppe Traina, Università di Catania.

2 La bibliografia su Ulisse è impossibile da riassumere in una noterella: cfr. almeno Boitani 2012².

3 Cfr. Consolo 1993 e Consolo, Nicolao 1999. 
Sull'ulissismo esplicito in Consolo esiste un bel saggio di Massimo Lollini nel quale quest'aspetto mi pare sia affrontato in modo quasi esaustivo. Riassumo i punti principali del discorso di Lollini:

I. Ulisse è eroe del Mediterraneo, ma la civilizzazione del Mediterraneo ha rivelato in sé tanto il volto della violenza quanto quello della cultura e dell'intelligenza: sicché Consolo, in tale direzione, ipervalorizza l'emblema omerico dell'olivo e dell'olivastro, ripetendolo in più luoghi e facendone il perno del suo libro più inclassificabile;

II. «il viaggio di Odisseo diventa la metafora che consente a Consolo di ritrovare nel mito e nella letteratura un senso drammatico e complesso dell'esistenza personale e collettiva, non più legato ad alcuna ideologia di progresso della civiltà mediterranea» (Lollini 2005: 25);

III. Ulisse è anche immagine dell'esilio «inappagato per l'infinito che lo pervade e lo proietta in una dimensione di alterità»: da questa alterità deriva il lavoro dello scrittore, un lavoro di scavo nel linguaggio alla ricerca di «una parvenza di umano» (Lollini 2005: 25);

IV. odissiaco è il viaggio in mare come esperienza di tipo sia storicamente determinato (economico), sia come avventura intellettuale, nel senso codificato da Dante nel XXVI canto dell'Inferno;

$\mathrm{V}$. il racconto omerico è diventato fondativo di un mito perché ha unito i tre elementi basilari della civilizzazione mediterranea: il viaggio, il mare, la guerra;

VI. odissiaca è la centralità della catabasi come luogo del riconoscimento del dolore, della paura di morire;

VII. la profezia di Tiresia ha messo al centro del destino di Ulisse l'erranza, l'esilio come «riconoscimento dell'alterità intrinseca nella condizione dell'Ulisse moderno», che Consolo studia «in una prospettiva di lunga durata» (Lollini 2005: 29): talché discendono dall'inquietudine ulissiaca non solo i suoi protagonisti più immediatamente riconoscibili in questa condizione, ma perfino i satanisti di Aleister Crowley e il barone Cicio di Nottetempo, casa per casa;

VIII. data l'importanza del precedente rappresentato dall'Iliade, poema della guerra, in Consolo l'Odissea diventa «un viaggio penitenziale, un nostos di espiazione» (Lollini 2005: 30) del sopravvissuto rispetto agli orrori della guerra, un po' come in Primo Levi. Dunque la meta del nostos non è la patria, ma l'esilio stesso, anche perché non si può avere nostalgia di una patria distrutta dalla tecnologia, come dimostra L'olivo e l'olivastro;

IX. Consolo è condannato all'erranza nei due poli opposti del disgusto: Milano e la Sicilia. L'erranza non è una scelta ma una condizione dolorosa dell'Ulisse moderno;

X. unica alternativa per Ulisse è il naufragio. Ovvero, nei termini consoliani, il naufragio del romanzo, l'afasia. Che è poi effetto 
della dimensione etica che distingue Consolo dal mero approccio solipsistico al presente (la disperazione);

XI. se l'Ulisse omerico ha messo in discussione il suo nome e la sua identità, chiamandosi Nessuno, l'Ulisse moderno riconosce la mancanza di identità come un valore perché apre all'ibridazione culturale (che è un vero e proprio topos del Consolo saggista tra gli anni Ottanta e Duemila, dunque con ampio e preveggente anticipo su temi che poi diventeranno vulgata culturale, almeno per buona parte della sinistra intellettuale italiana).

Fin qui Lollini. A integrazione della sua analisi si può ulteriormente sottolineare che la dimensione tragica (ma anche di pietas ${ }^{4}$ ) del discorso di Consolo, soprattutto dell'ultimo Consolo vieppiù odissiaco, non è soltanto riconducibile alla formazione classica, con netta preferenza per la cultura greca su quella latina, ma si innerva anche di contributi che sul piano strettamente letterario si rivolgono soprattutto alla cultura araba (con naturale predilezione per i poeti arabi di Sicilia) e dei paesi sudamericani o, in genere, delle culture postcoloniali. Mentre sul piano non letterario è sempre viva in Consolo l'attenzione alla cultura popolare siciliana. E qui si potrebbe innestare una riflessione su quello che Consolo considera popolare, perché mi pare che ci sia in lui, come c'è nella migliore tradizione letteraria siciliana, un netto rifiuto delle forme più arcaiche e arretrate della cultura popolare ${ }^{5}$ mentre c'è un interesse sempre vivo (e atipico, per un letterato italiano) nei confronti del popolo che vuole emanciparsi attraverso l'acculturazione (l'olivo) e anche verso la cultura tecnico-scientifica purché nella sua versione pre-tecnologica: si pensi alla botanica e all'arboricoltura che si affaccia in Retablo e di cui si fa vessillifero soprattutto lo zio/padre putativo di Chino Martinez nello Spasimo di Palermo. Queste aperture si sostanziano di una conoscenza perfino erudita di tutta una pubblicistica scientifica, soprattutto di ambito pre-novecentesco e siciliano; e anche queste aperture rientrano, a mio avviso, nella prospettiva ulissiaca in quanto si collegano alla dicotomia tra olivo e olivastro e anche in quanto considerano la techne non meccanizzata come una forma tra le più alte dell'intelletto umano e di una misura umana del vivere: si ricordi il grande emblema del letto nuziale costruito da Ulisse ricavandolo da un unico blocco di legno d'ulivo.

$\mathrm{Ci}$ sono però alcuni micro-elementi che rinviano a singoli punti della presenza odissiaca in Consolo e che non mi pare siano stati messi in evidenza a sufficienza. Per esempio, vorrei ricordare che, ben prima di Retablo, emerge il tema del viaggio, non massicciamente nella Ferita dell'aprile ma più chiaramente nel Sorriso dell'ignoto marinaio, e comunque sempre in posizione incipitaria:

4 Cfr. Turchetta 2015: LXVIII-LXXIV.

5 Leggiamo in Ratumemi: "Contra la forza nun cci vali la raggiuni” concluse il primo. E: "E basta, vah! Finiamola con 'sti proverbi vani degli antichi!"» (Consolo 2015: 534). 
«Dei primi due anni che passai a viaggiare mi rimane la strada arrotolata come un nastro, che posso svolgere» (Consolo 2015: 5) ${ }^{6}$ - ma è bene ricordare che nel primo romanzo il viaggio non si compie via mare: né questo di cui parla all'inizio, che gli serve a raggiungere la scuola, né quello in camion col padre: quest'ultimo, però, occupa un importante spazio nella Bildung dell'io narrante, sia perché lo lega al padre (figura positiva, alla stregua del vero padre di Consolo e a lui abbastanza somigliante, peraltro) sia perché gli permette di abbozzare un episodio nel bosco che rimanda all'importante racconto I linguaggi del bosco (che si legge nella seconda sezione delle Pietre di Pantalica). Anche il Sorriso dell'ignoto marinaio comincia all'insegna del viaggio con un clamoroso periodo nominale e proprio con le parole: «Viaggio in mare di Enrico Pirajno barone di Mandralisca da Lipari a Cefalù con la tavoletta del ritratto d'Antonello [...]» (Consolo 2015: 127).

A proposito di viaggi, forse si potrebbe ricondurre al modello dell'Odissea anche la struttura del viaggio 'a stazioni' ${ }^{7}$ molto presente nella narrativa consoliana: spesso si tratta di tappe previste, ma talvolta è il contrario e i personaggi si fermano a causa di rapine, fughe o accidenti vari; questa struttura è tipica della tavola centrale di Retablo e ovviamente anche dell'Olivo e l'olivastro (ma non è assente neppure nello Spasimo di Palermo) e appare in singoli momenti di altri testi, come nel caso dei due viaggi col padre a cui accennavo prima, o in Le vele apparivano a Mozia (Consolo 2012: 124-127), che racconta il viaggio a cui fu ispirato Retablo, o ancora in un racconto come La grande vacanza orientaleoccidentale (Consolo 2012: 163-169), che ci dà modo di risalire con molta chiarezza alla vocazione del giovane Consolo a viaggiare per la Sicilia e a leggerne l'identità all'insegna della grecità. Vocazione alla quale si giustapporrà, senza scacciarla, l'altra e successiva vocazione a valorizzare l'età dell'oro della Sicilia araba, «unico e irripetibile esempio di equilibrio ateniese» (Consolo 2015: 1215).

Non vanno sottovalutati anche taluni micro-aspetti ricondotti esplicitamente dall'autore a Omero: per esempio, quasi alla fine del quinto capitolo della Ferita dell'aprile, Filippo Mùstica si spoglia, salta in groppa al mulo ed entra in acqua per fare il bagno con l'animale. È una sequenza che ci verrebbe facile definire panica $^{8}$ ma che invece Consolo attribuisce a tutt'altra ispirazione, perché il ragazzo «tirò avanti come un condottiero, di quelli delle storie d'Omero» (Consolo 2015: 54). Dell'Iliade, insomma.

Ancora: all'inizio del capitolo $\mathrm{X}$ della Ferita dell'aprile, troviamo un intrigante paragone tra il mare e i campi coltivati:

6 Per inciso, un nastro arrotolato è abbastanza simile, come forma, a una chiocciola e ci pare quasi un'anticipazione del simbolo fondativo del Sorriso dell'ignoto marinaio.

7 E chissà che Consolo, attento lettore di Benjamin e di Enzensberger, non avesse letto anche Mittner e riflettuto sul modello dello Stationendrama o che l'abbia ripreso direttamente da Conversazione in Sicilia.

8 Forse anche dannunziana, se si potesse azzardare - e io lo farei - anche quest'aggettivo per definire certi aspetti della prosa consoliana. 
Le reti stese sopra la spiaggia parevano campi di terra grassa, orti concimati, tra la rena grigia. Il cielo scivolava fitto di qua dalle colline e sopra il mare verde e come scosso da un brivido si levava un tetto, spesso, che finiva lontano verso l'orizzonte, dov'era chiaro, lucente, come una lista di sole fra le stecche della gelosia: mare e cielo erano due piani paralleli, all'infinito. (Consolo 2015: 97).

Può essere utile ricordare, sempre nella logica dell'interesse di Consolo per le specificità della coltivazione agricola, quei punti, presenti in altre opere, in cui il nostro autore si sofferma sulla fertilità della terra: per esempio la terra grassa che si nasconde sotto la lumaca nel Sorriso dell'ignoto marinaio o quel bellissimo passo di Filosofiana in cui Vito Parlagreco comincia a zappare un campo pieno di quelli che noi chiameremmo reperti archeologici e che per lui non sono inutili cocci senza valore (come, invece, lo sono per gli abitanti di Mozia che guardano il cavaliere Clerici come un matto quando vuol pagare loro la statua dell'Efebo) ma «capricci» (Consolo 2015: 542) degli antichi, che però lo distraggono dall'occupazione primaria, cioè la coltivazione di quel gramo spicchio di terra. Perché, nonostante l'apparenza di inutile petraia, sotto questo strato di antichi cocci, Vito trova "una terra nera e grassa, terra di verginità immacolata» (Consolo 2015: 540). Se volessimo un po' estremizzare, potremmo perfino dire che non solo la natura è sotto la natura (la terra sotto la chiocciola), ma anche che sotto la cultura (che può anche essere percepita come sovrastruttura) c'è la natura, nella sua forma colturale ma anche, appunto, culturale: insomma, ci sono due forme dell'olivo ma non l'olivastro.

La bipartizione tra olivo e olivastro non è stata sempre così netta e dominante in Consolo: starei per dire 'fortunatamente', dato che a me sembra perfino un po' eccessiva nella sua formulazione così oppositiva. Si legge nella Ferita dell'aprile, in un passo in cui circola aria non soltanto siciliana ma anche greca, omerica:

così rugoso e scuro, così contorto, il carrubo l'avevo sempre pensato venuto dall'oscurità del tempo, che affondasse le radici al centro della terra: e il tronco mi pareva di tufo e i frutti di carbone. L'ulivo come il carrubo, la vite e l'asino e le capre, le lancelle di mosto e gli scifi di quagliata, certi vecchi accoccati ma saldi, mi parevano cose antichissime e immortali. (Consolo 2015: 111).

La conclusione della Ferita dell'aprile recita: "Così girai tanti anni per i paesi, all'isole, sulle montagne, alle marine, dal comune d'Alì fino a Messina» (Consolo 2015: 121). In pochissime parole, c'è quasi una panoramica, prima, sull'intero 'mondo' che il giovane siciliano impiegato del Dazio può immaginare e accogliere in una definizione vaga quanto vasta; poi, c'è la precisazione, che riduce tutto al vero 'mondo conosciuto', una porzione nemmeno troppo grande della provincia di Messina, che non è certo la più piccola tra le province siciliane: un allargamento della prospettiva geografica senza una chiara visione di quello che concretamente attende l'io narrante. Sarà certo ben più ampia la rinnovata prospettiva esistenziale del viaggiatore Petro Marano approdato in Tunisia alla conclusione di Nottetempo, casa per casa; ma ricordiamo che l'io narrante della Ferita dell'aprile prima viaggiava soltanto sui monti più vicini a casa e invece alla fine si slancia, con il nuovo lavoro che è frutto della sua educazione scolastica, 
alla conquista di un territorio sia montuoso che marittimo (l'autore lo specifica, volutamente). Dal canto suo, Petro non è ancora il viaggiatore che sperimenta la disperazione radicale dell'Olivo e l'olivastro e dello Spasimo di Palermo ${ }^{9}$, tant'è che rifiuta l'esilio in versione anarchica del compagno di viaggio Schicchi e si considera, costruttivamente, «solo come un emigrante, in cerca di lavoro, casa, di rispetto. Solo ad aspettare con pazienza che passasse la bufera» (Consolo 2015: 755).

Come ha ricordato Lollini, uno degli elementi basilari del viaggio di Ulisse è la catabasi: sorvolando sulla sensazione che per Consolo il vero viaggio all'inferno sia l'imbattersi nella sofferenza degli uomini nel mondo, nelle sue varie forme - una tra le più rilevanti è senz'altro la follia -, può essere interessante rilevare, a ulteriore conferma del ruolo tutto particolare giocato da Retablo nella storia letteraria del suo autore ${ }^{10}$, la continua alternanza metaforica tra inferno in terra e paradiso in terra. Quando Isidoro comincia a lavorare come facchino alla Cala dice: «Ero nell'inferno» (Consolo 2015: 373); quando, di ritorno dal viaggio, si aggira in cerca di Rosalia: "Mi parvi preso da' turchi, da' corsari, rapito in un sogno angustiante. Girai per vichi, per strade, per piazze, del Borgo, della Kalsa». Poi, dopo questo nuovo tuffo all'inferno, ascende al paradiso nell'oratorio di San Lorenzo: «Entrai, mi parve d'entrare in paradiso» (Consolo 2015: 375). E poi di nuovo in giro, «senza posa sulla strada, pazzo, forsennato» (Consolo 2015: 376). Si ritrova nell'intero romanzo, però, l'idea che tutta la realtà siciliana sia un'alternanza di inferno e paradiso: ancora Isidoro, durante il bagno termale a Segesta, "Ma qui è il paradiso!" sclamò Isidoro, più sempre in beatitudine o in ebetudine vagante» (Consolo 2015: 402). Allusioni all'inferno ci sono invece nel rivolgersi di Clerici alla Beccaria: «nulladimeno, pur sulla soglia di questa forte terra, nel primo cerchio di questo vortice di luce, sull'ingresso di questo laberinto degli olezzi, nell'incamminamento di questa galleria de' singolari tratti e d'occhi ardenti» (Consolo 2015: 403). La narrazione del viaggio da parte di Clerici è trasognata: «E a mano a mano io mi trovai a passare dal sogno e dall'incanto al risveglio più lucido, alla visione più netta delle cose, ne la luce di giugno più vere e crude, ch'invade l'animo mio d'incertezza e d'ansia pel futuro, finito questo tempo sospeso e irreale del viaggio» (Consolo 2015: 380-381); anche per lui, i facchini arrembanti tra la folla della Cala si presentano come invadenti e infernali, tanto più dopo la visione dei corpi degli impiccati e dei torturati. Ne deriva l'elezione di Isidoro come unica persona umile e istruita, che si distingue nel branco. In realtà Clerici, anche se porta con sé l'Odissea ${ }^{11}$, si presenta come un viaggiatore fin troppo diverso dall'Ulisse omerico. Ricordiamo la sua motivazione al viaggio:

9 Molto interessante l'interpretazione di Daragh O' Connell, che distingue tra la «disfatta» del personaggio e la «disperazione» dell'autore: cfr. O' Connell 2008: 174.

10 Cfr. Traina 2001 e Traina 2014.

11 Cfr. Consolo 2015: 406. 
volli sottrarmi alla tempesta insana d'ogni sentimento percorrendo a ritroso, per gli antichi sentieri della storia, questo tempo umano del conato, del movimento cieco e incontrollato, fino al punto oscuro iniziale per cui si passa nell'immota eternitate da cui veniamo, nel tempo senza soli e senza lune, giorni e stagioni, natività e morte, del vuoto e del silenzio, nell'immensa stasi, la somma e infinita quiete metafisica, nel modo come spiega il Campanella (Consolo 2015: 383).

Egli sembra proprio l'antitesi del viaggiatore, soprattutto del viaggiatore per mare; gli basta lo sballottamento d'un sentiero disagevole ed ecco il paragone enfatico: «E la lettica è come il maremoto, un guscio di barchetta sopra creste e avvalli delle onde, e i naviganti sbattuti e tormentati a dritta e a manca» (Consolo 2015: 385). Poi però impara dell'altro:

Poi domani, vicende sempre nuove, nuove avventure, ignote, che è l'essenza stessa della vita, che dentro i due certi punti, l'avvio e la sua fine, ricomincia l'avventura ogni mattina. E ancor di più l'essenza della vita dentro nel viaggio, per cui viaggio si fa dentro il viaggio, ignoto nell'ignoto. Così ora capisco coloro che viaggiano, capisco gli eterni erranti, i nomadi, i gitani: vivono ancor di più dei sedentari, dilatano il tempo, ingannano la morte (Consolo 2015: 440).

D'altronde non c'è differenza tra sogno, viaggio e scrittura di viaggio, per il cavaliere Clerici:

Mai sempre tuttavia il viaggio, come distacco, come lontananza dalla realtà che ci appartiene, è un sognare. E sognare è vieppiù lo scrivere, lo scriver memorando del passato come sospensione del presente, del viver quotidiano. E un sognare infine, in suprema forma, è lo scriver d'un viaggio, e d'un viaggio nella terra del passato. Come questo diario di viaggio che io per voi vado scrivendo, mia signora» (Consolo 2015: 413).

Infine, la prosecuzione del viaggio, verso la Spagna, forse il Nuovo Mondo, sembra dettata, di botto, soltanto dalla notizia del matrimonio di donna Teresa, non da altri motivi più consoni a un vero viaggiatore.

In Retablo troviamo altri episodi o motivi riconducibili all'Odissea. L'ospitalità di Soldano Lodovico potrebbe ricordare quella di Alcinoo; anche l'impressione che Clerici suscita al suo apparire - «e mi sentii, così chiaro di pelo, chiaro di pelle e panni, così alto e scarno, un'apparizione inusitata, un palo o baccalaro come bersaglio di tutte quell'occhiate dritte e acute della corte, oblique e gravi della loggia» (Consolo 2015: 388) - ricorda un po' l'apparizione di Ulisse a Nausicaa, dopo che si è lavato ma anche dopo che "Atena, la figlia di Zeus, lo fece / più grande e più robusto a vedersi» ${ }^{12}$. Ci sono dei paragoni espliciti: immergersi nei Bagni Segestani provoca

Una dimenticanza di sé, degli altri, di passioni e crucci, come i compagni d'Ulisse che nella terra dei Lotofagi mangiando il fior dolcissimo obliarono il ritorno o chi assunse il farmaco triste o altri doni accettò della magica Circe, o chi si perse ascoltando una volta il canto stregato di Sirene, come mi persi anch'io nel vacuo

12 Omero 2010: 407 (libro VI, vv. 229-230). 
smemorante, nel vago vorticare, nella felicità senza sorgente e nome» (Consolo 2015: 402).

Mentre nella palude di Mozia «annega la memoria: vana sembra la ricerca, la prova d'un racconto»: qui però la posta è più alta, è in gioco la possibilità stessa di narrare, come sfida di conoscenza all'ignoto: «Ma se appena ci volgiamo, castigo di sale, siamo spinti ancora, per lo scarto d'un riquadro, più dentro alla cascata, più presso alla montagna: e troveremo un varco, un passaggio, e cosa ci attende dietro quel sipario?» (Consolo 2015: 442). Nella storia dell'interpretazione del personaggio Ulisse molti si sono soffermati sul fatto che Ulisse è tanto personaggio narrato quanto personaggio narratore di sé, dunque, in qualche modo, controfigura archetipica del narratore, dello scrittore ${ }^{13}$.

Altri segni odissiaci in Retablo possono esserci anche per opposizione: Curàtolo Nino, «quell'uomo, alto e vigoroso, vestito delle pelli dei montoni» (Consolo 2015: 407), è un anti-Polifemo: ed è talmente anti-Polifemo da essere maestro d'inganni, quando suggerisce a Clerici di fingersi medico risanatore del mal di denti del brigante.

Si ricordi il ritornare, come basso continuo nell'opera di Consolo, di Morte per acqua di T.S. Eliot: una sorta di monito periodico, che riguarda il conflitto natura-cultura, oriente-occidente, terra-mare, poesia-commercio. Una specie di inceppo della fantasia, forse un incubo, forse il grande incubo di un greco abitatore di isole ${ }^{14}$. Ma sul motivo dell'isola bisognerebbe rileggere con attenzione almeno lo Spasimo di Palermo, dove si trovano diverse declinazioni, anche metaforiche, di tale tema: dagli «anni di serena gioia» in cui Chino e Lucia vivono «davanti al grandioso mare, all'isola che appare e che dispare all'orizzonte» (Consolo 2015: 928) giù giù fino all'isola, «che egli in cuore malediva, matrice d'ogni male, estremo lembo, perenne scivolio, annientamento», e infine a Enna, «isola sopra l'altipiano» (Consolo 2015: 931).

Lollini giustamente ha ricordato che il viaggio per mare è anche temuto dalla cultura greca, perché implica il rischio della morte per acqua senza seppellimento: in Retablo abbiamo una versione felicemente capovolta di questo timore nell'episodio dell'inabissamento della statua dell'Efebo di Mozia.

Dunque, il viaggio non promana soltanto il fascino intellettuale della sfida ai propri limiti (come in Dante), ma anche autentica paura. Tali ambivalenze riguardano anche il rapporto con la Natura di alcuni personaggi di Consolo: si pensi al tema del licantropismo che domina Nottetempo, casa per casa, o in Filosofiana al 'notturno' leopardiano di Vito Parlagreco, il quale, per la verità, s'interroga soprattutto sulla labilità della storia, ma poi - nel confronto con il pastorello mutangolo e con la lepre morta - viene profondamente inquietato dal tema funebre. Da lì a una riflessione sulla scrittura, il passo è breve:

$13 \mathrm{Ma}$, a tal proposito, si legga la finissima analisi di O' Connell, fondata sul confronto tra un passo dello Spasimo di Palermo e il suo avantesto Consolo 1995. Cfr. O' Connell 2008: 174-179.

14 Cfr. Curi 2005: 77-84. 
Solo che sogno o favola poco è diverso. Non è sogno tutto quanto si racconta, s'inventa o si riporta, per voce, per scrittura o in altro modo, d'una vicenda d'ieri, di oggi o di domani, d'una vicenda possibile o fantastica? È sempre sogno l'impresa del narrare, uno staccarsi dalla vera vita e vivere in un'altra. Sogno o forse anche una follia, perché dalla follia è proprio la vita che si stacca e che procede accanto, come ombra, fantàsima, illusione, all'altra che noi diciamo la reale. O della morte? (Consolo 2015: 545-546)

A proposito della grande metafora ulissiaca dell'erranza, va detto che Consolo la riserva, sì, a diversi suoi protagonisti ma, prima che essa trovi gli accenti tragici e non riconciliati dello Spasimo di Palermo (romanzo in cui sono in gioco davvero tante cose, forse troppe), la metafora dell'erranza mi pare che assuma tinte anche alquanto ambivalenti. Per esempio, se riflettiamo sulla componente autobiografica del tema dell'erranza o dell'esilio, si consideri che Consolo ha narrato in tante occasioni e dietro diversi schermi, finzionali o no, il suo arrivo a Milano, da studente universitario, e la scoperta che esisteva a Milano, non lontano dall'Università Cattolica dove studiava, una sorta di luogo concentrazionario di accoglienza e smistamento per gli emigranti meridionali. Molto onestamente, Consolo non ha mancato di sottolineare come, rispetto al destino animalesco degli emigrati poveri, la sua condizione di studente emigrante fosse piuttosto diversa: non un privilegiato, ma neppure un disperato, ammassato in transito verso altre destinazioni.

Ma sul tema dell'esilio abbiamo due ulteriori approfondimenti della prospettiva di Consolo: come abbiamo già visto, nell'Olivo e l'olivastro l'esilio è l'autoesilio, conseguenza del disgusto per l'invivibilità della Sicilia; nello Spasimo di Palermo il discorso da un lato si allarga alla tragedia civile della Sicilia infettata dalla mafia, dall'altro si restringe - ma quanto si restringe in profondità! Mi verrebbe da dire che si verticalizza verso il basso, nel romanzo del marabutto, della tragedia del nascondiglio ipogeo - a una prospettiva individuale, come dimostrano le parole conclusive del primo capitolo: «L'esilio è nella perdita, l'assenza, in noi l'oblio, la cieca indifferenza» (Consolo 2015: 890).

In uno scrittore come Consolo, l'influsso omerico non può mancare anche nelle scelte stilistiche. È sufficiente procedere un po' a casaccio, quasi ad apertura di libro, per trovare qualche esempio. Attingo alle Pietre di Pantalica per un riconoscibilissimo calco omerico come «E Filippo Siciliano [...] così parlò» (Consolo 2015: 520), che dà slancio epico a un personaggio di cui Consolo è stato amico e di cui ammirava la coerenza nella lotta per i diritti dei contadini; ma attingo allo stesso libro anche per un modulo spiccatamente narrativo (e che al nostro orecchio suona omerico) che è l'abbrivio epico al passato remoto, spesso seguito da complemento di luogo. Un solo esempio, tra i tanti possibili: «Mossero per via Bivona e Castelvecchio, su per la mulattiera» (Consolo 2015: 515). Weinrich ci ha insegnato che, se il passato remoto è il tempo grammaticale della storia, dell'epos, della guerra, delle vittorie e delle sconfitte, l'imperfetto durativo è il tempo dell'eterna e immutabile ciclicità; attingo da Malophòros: 
«S'adagiava, tutta l'area sacra, circondata da mura, sul fianco della collina» (Consolo 2015: 580); e, ancor più, alla fine del racconto:

A poco a poco non sentii più le parole di Tusa, guardavo Angelina e Ignazio, questi due giovani vecchi di ottant'anni passati, [...]. Persone reali, qui con me, su questa collina di Malophòros, in questo radioso mattino del primo giorno dell'anno, che pure a poco a poco svanivano, venivano prese in un vortice, precipitavano con me nel pozzo di Ecate, incontro alle divinità sotterranee, nel mistero e nell'oscurità infinita del Tempo. (Consolo 2015: 581)

Nell'economia delle Pietre di Pantalica, Malophòros è il racconto della quiete illusoria, delle accoglienze oneste e liete, dell'immersione nel mondo umano e civile di Selinunte ma anche in quello ctonio della Malophòros appunto: insomma, uno tra i racconti più sereni e rasserenanti che Consolo abbia scritto. Ma come dimenticare che questa quiete è continuamente insidiata dall'agitazione motoria di Ignazio, dell'inarrestabile Buttitta? E come non dire che al suo un po' eccezionale ritmo piano, alla sua quiete, quantitativamente Consolo preferisce l'agitazione dromomaniaca dei suoi protagonisti? Non solo il cavaliere Clerici, ma anche l'io saggistico di certe pagine delle Pietre di Pantalica (penso soprattutto a Comiso), l'io narrante dell'Olivo e l'olivastro, e soprattutto il povero Chino Martinez dello Spasimo di Palermo. In questi testi è tutto un andare e venire da un punto a un altro della Sicilia, o tra Milano, Parigi e la Sicilia, secondo tragitti - non sono sempre logici sul piano topografico ma dettati da un istinto che è soprattutto non dominabile istinto di fuga - che trovano il loro modulo espressivo preferito nel sintagma "Via da..." che ricorre tante di quelle volte che quasi non fa macchia nel testo; mentre non si può dimenticare, se non altro per l'inciampo semantico costituito dalla voce di origine dialettale e rara, quella sequenza di Retablo di identico significato ma che inizia con «Arrasso, arrasso, mia nobile signora, arrasso dalla Milano attiva» e si conclude circolarmente con "Arrasso dalla mia terra e dal mio tempo, via, via, lontan!» (Consolo 2015: 433), dove il cavaliere Clerici sembra recuperare in extremis con l'avverbio tronco la sua milanesità linguistica pesantemente revocata in gioco dall'uso del meridionalissimo 'arrasso' 15.

A questo punto, una piccola divagazione, sempre di segno odissiaco: proprio mentre si trova, e sappiamo che non è un caso, a Selinunte il cavaliere Clerici rifiuta la prospettiva del nostos a Milano, si dichiara felicemente prigioniero (anche dal punto di vista linguistico) della Sicilia che attraversa da ulisside. Bene, il rifiuto della Milano «attiva, mercatora» a me fa pensare non soltanto alle note prese di posizione antiborghesi di Consolo ma anche a un sottotesto che mi rifiuto di credere Consolo non conoscesse: ossia all'Ulisse di Tennyson, che poteva aver letto nella classica traduzione di Giovanni Pascoli, al rifiuto del nostos compiuto, del continuare a regnare su un'Itaca in cui gli abitanti sono in preda alla logica solo economica, alla necessità per questo Ulisse nauseato

15 Lessema il cui uso risale, in Consolo, già alla Ferita dell'aprile: cfr. O' Connell 2008: 182 , nota 59 . 
di ripartire verso l'ignoto e di affidare lo scettro a Telemaco (di lui più adatto a intendersi con i regnicoli imborghesiti). Mentre non sarà senza significato che nello Spasimo di Palermo sia il figlio a sperimentare la condizione dell'esilio: come dire che a Itaca non c'è più posto nemmeno per Telemaco, mentre a Ulisse-Chino Martinez è riservato non l'esilio in un luogo (Parigi) dove, di fatto, Mauro si è rifatta una vita, ma l'erranza.

Un paio di considerazioni finali.

Non ho ancora menzionato il rapporto tra i viaggi dei personaggi alter ego di Consolo e il viaggio di Goethe in Sicilia, perché la mia intenzione era quella di utilizzare il meno possibile le pagine saggistiche e concentrarmi sul Consolo narratore. Però il saggio raccolto in Di qua dal faro mi dà l'agio di ricordare che, mentre Consolo sottolinea il fatto che Goethe ha dimostrato, in Sicilia, di volere sfuggire al rischio di «varcare la soglia dell'ignoto» (Consolo 2015: 1220), Consolo, nei suoi romanzi, ha dimostrato di volere affrontare l'ignoto, i suoi fantasmi, il mondo ctonio che li contiene anche se non li placa, ma l'ha fatto utilizzando tutte le mistificazioni letterarie possibili: i mascheramenti, gli spostamenti, i veli e i filtri che gli venivano offerti dal gioco, appunto letterario, di specchi, reduplicazioni, metafore, simboli, rimescolamenti di carte e raddoppiamenti di alter ego. Tutte armi più che legittime del suo armamentario mentale che forse non è barocco soltanto in Retablo o in Lunaria: si pensi al fatto che il romanziere delle scritte del Sorriso dell'ignoto marinaio ha inserito una scritta anche nel testamentario Spasimo di Palermo, ed è, non a caso, l'iscrizione incisa sull'arco dell'ingresso alla grotta dei Beati Paoli, che recita «PER ARTE E PER INGANNO» (Consolo 2015: 910).

Se le armi del raddoppiamento e del nascondimento sono per l'autore più che legittime, anche il lettore è legittimato a tentare di smascherarle. E a chiedersi, per esempio, se il marabutto dello Spasimo di Palermo non sia anche un aleph, ovvero - come mi pare che il testo suggerisca - l'illuminazione su una verità sconcertante, appresa da «un libro raro, da sempre sognato, sul quale aveva oltremodo fantasticato, TOPOGRAPHIA E HISTORIA GENERAL DE ARGEL» (Consolo 2015: 954). A interrogarsi su «i sensi vari del nome marabutto» (Consolo 2015: 955): non solo un luogo-incubo il cui ricordo rinnova lo strazio di Lucia e di Chino, ma anche un uomo, prete della moschea o maestro del Corano, eremita santo o sacro pazzo: si ricordi che nella pagina proemiale in corsivo leggiamo un «T'assista l'eremita» (Consolo 2015: 877), dunque un auspicio che così viene a cadere; e si pensi, sia pure per un attimo e senza trarne illazione alcuna, alla follia di frate Agrippino o al male catubbo del luponario.

D'altra parte, lo sappiamo bene dal proemio, Chino Martinez è un'ennesima nuova versione del multiforme mito di Ulisse: è un Ulisse che arriva, sì, a Itaca ma trova la reggia vuota, senza la Penelope impazzita e morta, dunque non sa a chi svelare «il segreto che sta nelle radici» e, preda di questa tragedia, ha bisogno della calma per «ritrovare il nome tuo d'un tempo, il punto di partenza». Il nome, il punto: l'aleph, insomma. 
E siamo legittimati a chiederci, infine, perché nello Spasimo di Palermo Consolo fa morire Chino Martinez insieme a Borsellino e non insieme a Falcone. Domanda stupida? Non so, io me la sono posta solo adesso, ripensando al fatto che, nell'immaginario collettivo degli anni atroci che abbiamo vissuto - noi da spettatori, altri un po' meno -, il giudice Falcone aveva una 'presenza' simbolica e un carisma superiori a quelli di Borsellino. E allora, perché non lui ma Borsellino? Mi sono dato tre risposte, di ordine assai diverso: la prima, che spalmo sul piano della realtà, è che, se già la strage di Capaci era stata vissuta dall'opinione pubblica come un'enormità, l'omicidio di Borsellino, così immediatamente successivo, fu vissuto ancora peggio, come l'emblema di una misura ormai colma; la seconda risposta è che sarebbe apparsa una ben misera casualità il fatto che Chino Martinez sfrecciasse sull'autostrada contemporaneamente alla macchina di Falcone (però questa risposta funziona sul piano della coerenza narrativa e della verosimiglianza romanzesca, a dimostrazione del fatto che, anche in un'opera-limite come lo Spasimo di Palermo, Consolo doveva pur fare i conti con le regole e le tradizioni del romanzo, che invece non mancava mai di definire come un genere ormai defunto); la terza risposta non si colloca su un piano, ma s'inabissa nei sottoscala del profondo, o dell'inconscio, e riguarda il fatto che, mentre Falcone muore insieme alla moglie, Borsellino invece muore davanti agli occhi della madre. Della quale Chino aveva in precedenza immaginato la pena, l'ansia e, nella sua immaginazione, aveva pensato a come anche Lucia, se fosse sopravvissuta alla follia, avrebbe atteso con ansia e con pena il ritorno di Mauro. L'ansia e la pena, se si vuole, di Penelope. E già che siamo a questa trafila metaforica femminile, vale la pena di ricordare il bellissimo inizio del decimo capitolo, quando Chino stenta a ricordare i tratti del volto di Lucia, che pare poi trascolorare in Euridice («il volgere le spalle, l'andarsene man mano, lontana nel sacro regno, oscuro») ma anche, poco dopo, nella potentissima immagine omerica dell'impossibile abbraccio di Ulisse all'ombra della madre: «La piena assenza. Lui mùtilo, smarrito, perso nell'inconsistenza, nel protendere le braccia, stringere a sé un’ombra» (Consolo 2015: 958). La madre, dunque, arcana immagine - omerica e perenne - collegata alla morte.

\section{Bibliografia}

Boitani P., 2012², L'ombra di Ulisse. Figure di un mito, Bologna, il Mulino.

Consolo V., 1993, Fuga dall'Etna. La Sicilia e Milano, la memoria e la storia, a cura di Renato Nisticò, Roma, Donzelli.

Consolo V., 1995, I barboni, in: Sgubin: Opere 1988-1995, Milano, Electa.

Consolo V., 2012, La mia isola è Las Vegas, a cura di Nicolò Messina, Milano, Mondadori.

Consolo V., 2015, L'opera completa, a cura e con un saggio introduttivo di Gianni

Turchetta e uno scritto di Cesare Segre, Milano, Mondadori, «I Meridiani».

Consolo V., Nicolao M., 1999, Il viaggio di Odisseo, Milano, Bompiani. 
Curi U., 2005, «Uno, nessuno, molti: ritratto di Odisseo», Letture Classensi, $n^{\circ} 32-34,2005$, p. 77-84.

Lollini M., 2005, «Intrecci mediterranei. La testimonianza di Vincenzo Consolo, moderno Odisseo», Italica, LXXXII, n 1,2005 , p. 24-43.

O’Connell D., 2008, «Consolo narratore e scrittore palincestuoso», Quaderns d'Italià, 13, p. 161-184.

Omero, 2010, Odissea, traduzione di Vincenzo Di Benedetto, Milano, Rizzoli.

Traina G., 2001, Vincenzo Consolo, Fiesole, Cadmo.

Traina G., 2014, Siciliani ultimi? Tre studi su Sciascia, Bufalino, Consolo. E oltre, Modena, Mucchi.

Turchetta G., 2015, «Da un luogo bellissimo e tremendo», in: Consolo V., L'opera completa, a cura e con un saggio introduttivo di Gianni Turchetta e uno scritto di Cesare Segre, Milano, Mondadori, «I Meridiani», p. XXIII-LXXIV. 